\title{
Existence and multiplicity of positive solutions for a class of fractional differential equations with three-point boundary value conditions
}

\author{
Bingxian $\mathrm{Li}^{1}$, Shurong Sun ${ }^{1 *}$, Ping Zhao ${ }^{2}$ and Zhenlai Han ${ }^{1}$
}

"Correspondence:
sshrong@163.com
'School of Mathematical Sciences,
University of Jinan, Jinan, Shandong
250022, P.R. China
Full list of author information is
available at the end of the article

available at the end of the article

\begin{abstract}
In this paper, we consider the nonlinear three-point boundary value problem of fractional differential equations

$$
D_{0^{+}}^{\alpha} u(t)+a(t) f(t, u(t))=0, \quad 0<t<1,2<\alpha \leq 3
$$

with boundary conditions

$$
u(0)=0, \quad D_{0^{+}}^{\beta} u(0)=0, \quad D_{0^{+}}^{\beta} u(1)=b D_{0^{+}}^{\beta} u(\xi), \quad 1 \leq \beta \leq 2,
$$

involving Riemann-Liouville fractional derivatives $D_{0^{+}}^{\alpha}$ and $D_{0^{+}}^{\beta}$, where $a(t)$ maybe singular at $t=0$ or $t=1$. We use the Banach contraction mapping principle and the Leggett-Williams fixed point theorem to obtain the existence and uniqueness of positive solutions and the existence of multiple positive solutions. We investigate the above fractional differential equations without many preconditions by the fixed point index theory and obtain the existence of a single positive solution. Some examples are given to show the applicability of our main results.
\end{abstract}

MSC: $34 \mathrm{A08} ; 34 \mathrm{~B} 18$

Keywords: fractional differential equations; three-point boundary value problem; existence and multiplicity; fixed point theorem; positive solution

\section{Introduction}

The development of fractional differential equations is accompanied by fractional calculus; see [1-6]. With wide applications of fractional calculus in the fields of physical, biological, heat conduction, chemical physics, economics, etc., the study of boundary value problems of fractional differential equations and inclusions gradually become hot issues for many mathematicians [7-14].

In recent years, some researchers focused themselves on the solutions, especially positive solutions of multipoint boundary value problems of fractional differential equations [15-20].

(c) $2015 \mathrm{Li}$ et al. This article is distributed under the terms of the Creative Commons Attribution 4.0 International License (http://creativecommons.org/licenses/by/4.0/), which permits unrestricted use, distribution, and reproduction in any medium, provided you give appropriate credit to the original author(s) and the source, provide a link to the Creative Commons license, and indicate if changes were made. 
In 2010, Li et al. [15] considered the existence and uniqueness for nonlinear fractional differential equation of the type

$$
D_{0^{+}}^{\alpha} u(t)+f(t, u(t))=0, \quad 0<t<1,1<\alpha \leq 2,
$$

subject to the boundary conditions

$$
u(0)=0, \quad D_{0^{+}}^{\beta} u(1)=a D_{0^{+}}^{\beta} u(\xi), \quad 0 \leq \beta \leq 1,
$$

where $D_{0^{+}}^{\alpha}$ is the standard Riemann-Liouville fractional-order derivative. They obtained the existence and multiplicity results of positive solutions by using some fixed point theorems.

In 2012, Jiang et al. [17] discussed the existence of positive solutions for a multipoint boundary value problem of the fractional differential equation

$$
\begin{aligned}
& D_{0^{+}}^{\alpha} u(t)+f(t, u(t))=0, \quad 0<t<1,1<\alpha \leq 2, \\
& u(0)=0, \quad D_{0^{+}}^{\beta} u(1)=\sum_{i=1}^{m-2} b_{i} D_{0^{+}}^{\beta} u\left(\xi_{i}\right), \quad 0<\beta<1,
\end{aligned}
$$

where $D_{0^{+}}^{\alpha}$ and $D_{0^{+}}^{\beta}$ are the Riemann-Liouville fractional derivatives. By fixed point index theory they obtained the existence results.

In 2013, Ahmad et al. [20] investigated the existence theory for nonlinear fractional differential equations of the type

$$
{ }^{c} D^{q} x(t)=f(t, x(t)), \quad 0<t<T, n-1<q \leq n, n \geq 2,
$$

subject to the boundary conditions

$$
x(0)=0, \quad x^{\prime}(0)=0, \quad \ldots, \quad x^{(n-2)}(0)=0, \quad x(T)=\sum_{i=1}^{m} \gamma_{i}\left(I^{\beta_{i}} x\left(\eta_{i}\right)-I^{\beta_{i}} x\left(\zeta_{i}\right)\right),
$$

where ${ }^{c} D^{q}$ are the Caputo fractional derivatives. Their results were based on some standard tools of fixed point theory, and they obtained the existence theory for nonlinear fractional differential equations of arbitrary order involving nonintersecting finitely many strips of arbitrary length.

Motivated by excellent results and the methods in [15, 17], in this paper, we investigate the three-point boundary value problem for the fractional differential equation

$$
\begin{aligned}
& D_{0^{+}}^{\alpha} u(t)+a(t) f(t, u(t))=0, \quad 0<t<1, \\
& u(0)=0, \quad D_{0^{+}}^{\beta} u(0)=0, \quad D_{0^{+}}^{\beta} u(1)=b D_{0^{+}}^{\beta} u(\xi),
\end{aligned}
$$

where $D_{0^{+}}^{\alpha}$ and $D_{0^{+}}^{\beta}$ are the Riemann-Liouville fractional derivatives, $2<\alpha \leq 3,1 \leq \beta \leq 2$, $\alpha \geq \beta+1,0<b<1,0<\xi<1, A:=1-b \xi^{\alpha-\beta-1}>0$, and $a(t)$ maybe singular at $t=0$ or $t=1$. We will systematically study the existence, uniqueness, and multiplicity of positive solutions to the three-point boundary value problem (1.1)-(1.2). 
The main innovation points of this paper are as follows. (i) Compared with [15], we propose some techniques to prove the complete continuity of operators and the properties of the Green function that need not add more premise conditions. (ii) Compared with [15], the properties of the Green function happen to change with the order of original equations increasing. We use these new properties to obtain more general results. (iii) In [15], to prove the existence of the solutions, the function of two variables $f(t, x)$ was imposed to some preconditions. In this article, we investigate the fractional differential equations without many preconditions by the fixed point index theory and obtain the existence of single positive solutions. In fact, the fractional differential equations can respond better to impersonal law, so it is very important to weaken preconditions. This work is motivated by the [17]. (iv) Compared with [17], we not only obtained the existence of solutions, but also obtained the uniqueness and multiplicity of solutions with three-point boundary value conditions. Our results extend some known results.

The plan of this paper is as follows. In Section 2, we shall give some definitions and lemmas to prove our main results. In Section 3, we establish the existence and uniqueness of single positives solutions to the three-point boundary value problem (1.1)-(1.2) by the Banach contraction mapping principle and the fixed point index theory, and we investigate the existence of multiple positives solutions for (1.1) and (1.2) by the Leggett-Williams fixed point theorem. In Section 4, we present examples to illustrate the main results.

In order to facilitate to our study, we make the following assumptions:

$\left(\mathrm{H}_{1}\right) f:[0,1] \times[0, \infty) \rightarrow[0, \infty)$ is a continuous function;

$\left(\mathrm{H}_{2}\right) a(\cdot) \in L^{1}(0,1)$ is nonnegative, $a(t)$ does not vanish identically on any subinterval of $[0,1]$, and $0<\int_{0}^{1} a(s)(1-s)^{\alpha-\beta-1} s^{\alpha-1} d s<\infty$.

\section{Preliminaries}

For the convenience of the reader, we present some necessary definitions and lemmas from the fractional calculus theory.

Definition 2.1 ([4]) The fractional integral of order $\alpha(\alpha>0)$ of a function $f:(0,+\infty) \rightarrow \mathbb{R}$ is given by

$$
I_{0^{+}}^{\alpha} f(t)=\frac{1}{\Gamma(\alpha)} \int_{0}^{t} \frac{f(s)}{(t-s)^{1-\alpha}} d s
$$

where $\Gamma(\cdot)$ is the gamma function, provided that the right side is pointwise defined on $(0,+\infty)$.

Definition 2.2 ([4]) The Riemann-Liouville fractional derivative of order $\alpha>0$ of a continuous function $f:(0,+\infty) \rightarrow \mathbb{R}$ is given by

$$
D_{0^{+}}^{\alpha} f(t)=\frac{1}{\Gamma(n-\alpha)}\left(\frac{d}{d t}\right)^{n} \int_{0}^{t}(t-s)^{n-\alpha-1} f(s) d s
$$

where $\Gamma(\cdot)$ is the gamma function, provided that the right side is pointwise defined on $(0,+\infty)$, and $n=[\alpha]+1$ with $[\alpha]$ standing for the largest integer less than $\alpha$.

Definition 2.3 ([4]) Let $E$ be a real Banach space. A nonempty closed convex set $K \subset E$ is called cone if 
(1) if $x \in K$ and $\lambda>0$, then $\lambda x \in K$;

(2) if $x \in K$ and $-x \in K$, then $x=0$.

Definition 2.4 ([15]) A map $\phi$ is said to be a nonnegative continuous concave functional on a cone $\mathrm{K}$ of a real Banach space $\mathrm{E}$ if $\phi: K \rightarrow[0, \infty)$ is continuous and

$$
\phi(\lambda x+(1-\lambda) y) \geq \lambda \phi(x)+(1-\lambda) \phi(y)
$$

for all $x, y \in K$ and $0 \leq \lambda \leq 1$.

Lemma 2.1 ([4]) Let $\alpha>-1, \beta>0$, and $t>0$. Then

$$
D_{0^{+}}^{\beta} t^{\alpha}=\frac{\Gamma(\alpha+1)}{\Gamma(\alpha-\beta+1)} t^{\alpha-\beta} .
$$

Lemma $2.2([4])$ Assume that $u(t) \in C(0,1) \cap L(0,1)$ and $D_{0^{+}}^{\alpha} u \in C(0,1) \cap L(0,1)$ with the Riemann-Liouville fractional derivative of order $\alpha>0$. Then

$$
I_{0^{+}}^{\alpha} D_{0^{+}}^{\alpha} u(t)=u(t)+c_{1} t^{\alpha-1}+c_{2} t^{\alpha-2}+\cdots+c_{N} t^{\alpha-N}
$$

where $c_{i} \in R, i=1,2, \ldots, N$, and $N$ is the smallest integer greater than or equal to $\alpha$.

Lemma 2.3 For Riemann-Liouville fractional derivatives, we have

$$
D_{0^{+}}^{\beta} \int_{0}^{t}(t-s)^{\alpha-1} f(s) d s=\frac{\Gamma(\alpha)}{\Gamma(\alpha-\beta)} \int_{0}^{t}(t-s)^{\alpha-\beta-1} f(s) d s,
$$

where $f \in L^{1}(0,1)$, and $\alpha, \beta$ are two constants with $\alpha-\beta-1 \geq 0$.

Proof From

$$
D_{0^{+}}^{\alpha} I_{0^{+}}^{\alpha} f(t)=f(t), \quad I_{0^{+}}^{\alpha} I_{0^{+}}^{\beta} f(t)=I_{0^{+}}^{\alpha+\beta} f(t)
$$

we get

$$
\begin{aligned}
D_{0^{+}}^{\beta} \int_{0}^{t}(t-s)^{\alpha-1} f(s) d s & =D_{0^{+}}^{\beta} \Gamma(\alpha) \frac{1}{\Gamma(\alpha)} \int_{0}^{t}(t-s)^{\alpha-1} f(s) d s \\
& =D_{0^{+}}^{\beta} \Gamma(\alpha) I_{0^{+}}^{\alpha} f(t)=\Gamma(\alpha) D_{0^{+}}^{\beta} I_{0^{+}}^{\alpha} f(t) \\
& =\Gamma(\alpha) D_{0^{+}}^{\beta} I_{0^{+}}^{\beta} I_{0^{+}}^{\alpha-\beta} f(t)=\Gamma(\alpha) I_{0^{+}}^{\alpha-\beta} f(t) \\
& =\Gamma(\alpha) \frac{1}{\Gamma(\alpha-\beta)} \int_{0}^{t}(t-s)^{\alpha-\beta-1} f(s) d s .
\end{aligned}
$$

The proof is completed.

The following lemma is fundamental in the proofs of our main results.

Lemma 2.4 (Banach contraction mapping principle [6]) Let $(X, d)$ be a nonempty complete metric space, and let $T: X \rightarrow X$ be a contraction, i.e., there exists a number $0 \leq \rho<1$ 
such that

$$
d(T x, T y) \leq \rho d(x, y) .
$$

Then the operator $T$ has a unique fixed point $x^{*} \in X$.

Lemma 2.5 (Krein-Rutman [6]) Let $K$ be a reproducing cone in a real Banach space E, and $L: E \rightarrow E$ be a compact linear operator with $L(K) \subseteq K$ and spectral radius $r(L)$. If $r(L)>0$, then there exists $\varphi \in K \backslash\{0\}$ such that $L \varphi=r(L) \varphi$.

Lemma 2.6 (Fixed point index theory [6]) Assume that $E$ is a Banach space, $K \subset E$ is a cone, and $\Omega(K)$ is a bounded open subset in $K$. Furthermore, assume that $T: \overline{\Omega(K)} \rightarrow K$ is a completely continuous operator. Then the following conclusions hold:

(i) If there exists $u_{0} \in K \backslash\{0\}$ such that $u \neq T u+\lambda u_{0}$ for all $u \in \partial \Omega(K)$ and $\lambda \geq 0$, then the fixed point index $i(T, \Omega(K), K)=0$;

(ii) If $0 \in \Omega(K)$ and $T u \neq \lambda u$ for all $u \in \partial \Omega(K)$ and $\lambda \geq 1$, then the fixed point index $i(T, \Omega(K), K)=1$.

Lemma 2.7 (Leggett-Williams fixed point theorem [6]) Let $K$ be a cone in a real Banach space $E, K_{c}=\{x \in K:\|x\|<c\}, \phi$ be a nonnegative continuous concave functional on $K$ such that $\phi(x) \leq\|x\|$ for all $x \in \bar{K}_{c}$, and $K\left(\phi, b_{1}, d\right)=\left\{x \in K: b_{1} \leq \phi(x),\|x\| \leq d\right\}$. Suppose that $T: \bar{K}_{c} \rightarrow \bar{K}_{c}$ is completely continuous and there exist positive constants $0<a<b_{1}<d \leq c$ such that

(i) $\left\{x \in K\left(\phi, b_{1}, d\right): \phi(x)>b_{1}\right\} \neq \emptyset$ and $\phi(T x)>b_{1}$ for $x \in K\left(\phi, b_{1}, d\right)$,

(ii) $\|T x\|<$ a for $x \in \bar{K}_{a}$,

(iii) $\phi(T x)>b_{1}$ for $x \in K\left(\phi, b_{1}, c\right)$ with $\|T x\|>d$.

Then $T$ has at least three fixed points $x_{1}, x_{2}$, and $x_{3}$ with $\left\|x_{1}\right\|<a, b_{1}<\phi\left(x_{2}\right)$, and $a<\left\|x_{3}\right\|$ with $\phi\left(x_{3}\right)<b_{1}$.

Remark 2.1 If $d=c$, then condition (i) implies conditions (iii).

\section{Main results}

Let $E=C[0,1]$ be a Banach space of all continuous real functions on $[0,1]$ endowed with norm $\|u\|=\sup _{0 \leq t \leq 1}|u(t)|$, and $K$ be the cone

$$
K=\{u \in E: u(t) \geq 0, t \in[0,1]\}
$$

Obviously, $K$ is a reproducing cone of $E$.

Let the nonnegative continuous concave functional $\phi$ on the cone $K$ be defined by

$$
\phi(u)=\min _{\xi \leq t \leq 1}|u(t)| .
$$

Lemma 3.1 Let $g(t) \in C(0,1) \cap L^{1}(0,1)$. Then the boundary value problem of the fractional differential equation

$$
\begin{aligned}
& D_{0^{+}}^{\alpha} u(t)+g(t)=0, \quad 0<t<1,2<\alpha \leq 3, \\
& u(0)=0, \quad D_{0^{+}}^{\beta} u(0)=0, \quad D_{0^{+}}^{\beta} u(1)=b D_{0^{+}}^{\beta} u(\xi), \quad 1 \leq \beta \leq 2,
\end{aligned}
$$


has the unique solution

$$
\begin{aligned}
u(t)= & -\int_{0}^{t} \frac{1}{\Gamma(\alpha)}(t-s)^{\alpha-1} g(s) d s+\frac{t^{\alpha-1}}{A \Gamma(\alpha)} \int_{0}^{1}(1-s)^{\alpha-\beta-1} g(s) d s \\
& -\frac{t^{\alpha-1}}{A \Gamma(\alpha)} \int_{0}^{\xi} b(\xi-s)^{\alpha-\beta-1} g(s) d s \\
= & \int_{0}^{1} G(t, s) g(s) d s,
\end{aligned}
$$

where

$$
G(t, s)= \begin{cases}\frac{\alpha^{\alpha-1}(1-s)^{\alpha-\beta-1}-b t^{\alpha-1}(\xi-s)^{\alpha-\beta-1}-A(t-s)^{\alpha-1}}{A \Gamma(\alpha)}, & 0 \leq s \leq \min \{t, \xi\}<1, \\ \frac{\alpha^{\alpha-1}(1-s)^{\alpha-\beta-1}-A(t-s)^{\alpha-1}}{A \Gamma(\alpha)}, & 0<\xi \leq s \leq t \leq 1, \\ \frac{\alpha^{\alpha-1}(1-s)^{\alpha-\beta-1}-b t^{\alpha-1}(\xi-s)^{\alpha-\beta-1}}{A \Gamma(\alpha)}, & 0 \leq t \leq s \leq \xi<1, \\ \frac{t^{\alpha-1}(1-s)^{\alpha-\beta-1}}{A \Gamma(\alpha)}, & \max \{t, \xi\} \leq s \leq 1 .\end{cases}
$$

Proof In view of Definition 2.1 and Lemma 2.2, it is clear that equation (3.1) is equivalent to the integral form

$$
u(t)=-I_{0^{+}}^{\alpha} g(t)+c_{1} t^{\alpha-1}+c_{2} t^{\alpha-2}+c_{3} t^{\alpha-3}
$$

for some $c_{1}, c_{2}, c_{3} \in R$. Consequently, the general solution of equation (3.1) is

$$
u(t)=-\int_{0}^{t} \frac{1}{\Gamma(\alpha)}(t-s)^{\alpha-1} g(s) d s+c_{1} t^{\alpha-1}+c_{2} t^{\alpha-2}+c_{3} t^{\alpha-3} .
$$

By the boundary condition $u(0)=0$ we find that $c_{3}=0$. In view of Lemma 2.3 and $D_{0^{+}}^{\beta} u(0)=0$, we have

$$
D_{0^{+}}^{\beta} u(t)=D_{0^{+}}^{\beta}\left(-\int_{0}^{t} \frac{1}{\Gamma(\alpha)}(t-s)^{\alpha-1} g(s) d s\right)+c_{1} \frac{\Gamma(\alpha)}{\Gamma(\alpha-\beta)} t^{\alpha-\beta-1}+c_{2} \frac{\Gamma(\alpha-1)}{\Gamma(\alpha-\beta-1)} t^{\alpha-\beta-2} .
$$

For $\alpha \in(2,3]$ and $\beta \in[1,2]$ such that $\alpha \geq \beta+1$, we have $\alpha-\beta-2 \in[-1,0]$. Thus, $c_{2}=0$. From

$$
D_{0^{+}}^{\beta} u(1)=b D_{0^{+}}^{\beta} u(\xi)
$$

we get

$$
c_{1}=\frac{1}{A \Gamma(\alpha)} \int_{0}^{1}(1-s)^{\alpha-\beta-1} g(s) d s-\frac{b}{A \Gamma(\alpha)} \int_{0}^{\xi}(\xi-s)^{\alpha-\beta-1} g(s) d s .
$$

Then the boundary value problem has the unique solution

$$
\begin{aligned}
u(t)= & -\int_{0}^{t} \frac{1}{\Gamma(\alpha)}(t-s)^{\alpha-1} g(s) d s+\frac{t^{\alpha-1}}{A \Gamma(\alpha)} \int_{0}^{1}(1-s)^{\alpha-\beta-1} g(s) d s \\
& -\frac{b t^{\alpha-1}}{A \Gamma(\alpha)} \int_{0}^{\xi}(\xi-s)^{\alpha-\beta-1} g(s) d s=\int_{0}^{1} G(t, s) g(s) d s .
\end{aligned}
$$

The proof is completed. 
Define the operator $T: K \rightarrow E$ and the linear operator $L: K \rightarrow K$ as follows:

$$
\begin{aligned}
T u(t)= & -\int_{0}^{t} \frac{1}{\Gamma(\alpha)} a(s)(t-s)^{\alpha-1} f(s, u(s)) d s+\frac{t^{\alpha-1}}{A \Gamma(\alpha)} \int_{0}^{1} a(s)(1-s)^{\alpha-\beta-1} f(s, u(s)) d s \\
& -\frac{b t^{\alpha-1}}{A \Gamma(\alpha)} \int_{0}^{\xi} a(s)(\xi-s)^{\alpha-\beta-1} f(s, u(s)) d s \\
= & \int_{0}^{1} G(t, s) a(s) f(s, u(s)) d s, \\
L u(t)= & \int_{0}^{1} G(t, s) a(s) u(s) d s .
\end{aligned}
$$

Set $g(t)=a(t) f(t, u(t))$ in Lemma 3.1. We deduce that $u$ is a solution of the boundary value problem (1.1)-(1.2) if and only if it is a fixed point of the operator $T$.

Remark 3.1 $a(t)$ may be singular at $t=0$ or $t=1$.

Lemma 3.2 The function $G(t, s)$ in Lemma 3.1 satisfies the following properties:

(i) $G(t, s)$ is continuous on $[0,1] \times[0,1]$;

(ii) $G(t, s)>0$ for any $t, s \in(0,1)$;

(iii) $G(t, s) \leq G(1, s)$, for any $t, s \in(0,1)$;

(iv) there exists a positive function $\gamma(s) \in C(0,1)$ such that

$$
\min _{\xi \leq t \leq 1} G(t, s) \geq \gamma(s) \max _{0 \leq t \leq 1} G(t, s)=\gamma(s) G(1, s), \quad 0<s<1 .
$$

Proof It is easy to see that (i) holds. So we prove that the rest are true. Let

$$
\begin{aligned}
& g_{1}(t, s)=\frac{t^{\alpha-1}(1-s)^{\alpha-\beta-1}-b t^{\alpha-1}(\xi-s)^{\alpha-\beta-1}-A(t-s)^{\alpha-1}}{A \Gamma(\alpha)}, \quad 0 \leq s \leq \min \{t, \xi\}<1, \\
& g_{2}(t, s)=\frac{t^{\alpha-1}(1-s)^{\alpha-\beta-1}-A(t-s)^{\alpha-1}}{A \Gamma(\alpha)}, \quad 0<\xi \leq s \leq t \leq 1 \\
& g_{3}(t, s)=\frac{t^{\alpha-1}(1-s)^{\alpha-\beta-1}-b t^{\alpha-1}(\xi-s)^{\alpha-\beta-1}}{A \Gamma(\alpha)}, \quad 0 \leq t \leq s \leq \xi<1, \\
& g_{4}(t, s)=\frac{t^{\alpha-1}(1-s)^{\alpha-\beta-1}}{A \Gamma(\alpha)}, \quad \max \{t, \xi\} \leq s \leq 1 .
\end{aligned}
$$

We will first show that

$$
g_{1}(t, s)>0, \quad 0 \leq s \leq \min \{t, \xi\}<1 .
$$

Since

$$
\begin{aligned}
g_{1}(t, s)= & \frac{1}{A \Gamma(\alpha)}\left(t^{\alpha-1}(1-s)^{\alpha-\beta-1}-b t^{\alpha-1}(\xi-s)^{\alpha-\beta-1}-A(t-s)^{\alpha-1}\right) \\
= & \frac{1}{A \Gamma(\alpha)}\left(t^{\alpha-1}\left(1-b \xi^{\alpha-\beta-1}+b \xi^{\alpha-\beta-1}\right)(1-s)^{\alpha-\beta-1}-b t^{\alpha-1} \xi^{\alpha-\beta-1}\left(1-\frac{s}{\xi}\right)^{\alpha-\beta-1}\right. \\
& \left.-A t^{\alpha-1}\left(1-\frac{s}{t}\right)^{\alpha-1}\right)
\end{aligned}
$$


Li et al. Advances in Difference Equations ( 2015) 2015:383

Page 8 of 19

$$
\begin{aligned}
= & \frac{1}{A \Gamma(\alpha)}\left(t^{\alpha-1}\left(A+b \xi^{\alpha-\beta-1}\right)(1-s)^{\alpha-\beta-1}-b t^{\alpha-1} \xi^{\alpha-\beta-1}\left(1-\frac{s}{\xi}\right)^{\alpha-\beta-1}\right. \\
& \left.-A t^{\alpha-1}\left(1-\frac{s}{t}\right)^{\alpha-1}\right) \\
= & \frac{1}{A \Gamma(\alpha)}\left(A t^{\alpha-1}\left((1-s)^{\alpha-\beta-1}-\left(1-\frac{s}{t}\right)^{\alpha-1}\right)\right. \\
& \left.+b t^{\alpha-1} \xi^{\alpha-\beta-1}\left((1-s)^{\alpha-\beta-1}-\left(1-\frac{s}{\xi}\right)^{\alpha-\beta-1}\right)\right) \\
> & \frac{1}{A \Gamma(\alpha)}\left(A t^{\alpha-1}\left((1-s)^{\alpha-1}-\left(1-\frac{s}{t}\right)^{\alpha-1}\right)\right. \\
& \left.+b t^{\alpha-1} \xi^{\alpha-\beta-1}\left((1-s)^{\alpha-\beta-1}-\left(1-\frac{s}{\xi}\right)^{\alpha-\beta-1}\right)\right) \\
> & \frac{1}{A \Gamma(\alpha)}\left(A t^{\alpha-1}\left((1-s)^{\alpha-1}-(1-s)^{\alpha-1}\right)\right. \\
& \left.+b t^{\alpha-1} \xi^{\alpha-\beta-1}\left((1-s)^{\alpha-\beta-1}-(1-s)^{\alpha-\beta-1}\right)\right) \\
= & 0,
\end{aligned}
$$

we deduce

$$
g_{1}(t, s)>0, \quad 0 \leq s \leq \min \{t, \xi\}<1 .
$$

By a similar argument we can conclude that

$$
g_{2}(t, s)>0, \quad 0<\xi \leq s \leq t \leq 1, \quad g_{3}(t, s)>0, \quad 0 \leq t \leq s \leq \xi<1,
$$

and

$$
g_{4}(t, s)>0, \quad 0 \leq \max \{t, \xi\} \leq s \leq 1
$$

Therefore, we get that $G(t, s)>0$ for any $s, t \in(0,1)$.

Next, we will prove (iii) is true. It is easy to check that $g_{3}(t, s)$ and $g_{4}(t, s)$ are increasing with respect to $t$ on $[0, s]$. We will show that $g_{1}(t, s)$ and $g_{2}(t, s)$ are increasing with respect to $t$ on $[s, 1]$.

Let $h_{1}(t, s)=g_{1}(t, s) \Gamma(\alpha)$. Then we have

$$
h_{1}(t, s)=\frac{t^{\alpha-1}(1-s)^{\alpha-\beta-1}-b t^{\alpha-1}(\xi-s)^{\alpha-\beta-1}-A(t-s)^{\alpha-1}}{A}, \quad 0 \leq s \leq \min \{t, \xi\}<1,
$$

and

$$
\begin{aligned}
\frac{\partial h_{1}(t, s)}{\partial t}= & \frac{(\alpha-1) t^{\alpha-2}}{A}\left((1-s)^{\alpha-\beta-1}-b \xi^{\alpha-\beta-1}\left(1-\frac{s}{\xi}\right)^{\alpha-\beta-1}-A\left(1-\frac{s}{t}\right)^{\alpha-2}\right) \\
= & \frac{(\alpha-1) t^{\alpha-2}}{A}\left(A\left((1-s)^{\alpha-\beta-1}-\left(1-\frac{s}{t}\right)^{\alpha-2}\right)\right. \\
& \left.+b \xi^{\alpha-\beta-1}\left((1-s)^{\alpha-\beta-1}-\left(1-\frac{s}{\xi}\right)^{\alpha-\beta-1}\right)\right)
\end{aligned}
$$




$$
\begin{aligned}
& >\frac{(\alpha-1) t^{\alpha-2}}{A}\left(A\left((1-s)^{\alpha-2}-\left(1-\frac{s}{t}\right)^{\alpha-2}\right)\right. \\
& \left.+b \xi^{\alpha-\beta-1}\left((1-s)^{\alpha-\beta-1}-\left(1-\frac{s}{\xi}\right)^{\alpha-\beta-1}\right)\right) \\
& >\frac{(\alpha-1) t^{\alpha-2}}{A}\left(A\left((1-s)^{\alpha-2}-(1-s)^{\alpha-2}\right)\right. \\
& \left.+b \xi^{\alpha-\beta-1}\left((1-s)^{\alpha-\beta-1}-(1-s)^{\alpha-\beta-1}\right)\right) \\
& =0 \text {. }
\end{aligned}
$$

So we have $\frac{\partial h_{1}(t, s)}{\partial t}>0$, and thus $g_{1}(t, s)$ is increasing with respect to $t$ on $[s, 1]$.

Next, we show that $g_{2}(t, s)$ is increasing with respect to $t$ on $[s, 1]$.

Let $h_{2}(t, s)=g_{2}(t, s) \Gamma(\alpha)$. Then we have

$$
h_{2}(t, s)=\frac{t^{\alpha-1}(1-s)^{\alpha-\beta-1}-A(t-s)^{\alpha-1}}{A}, \quad 0<\xi \leq s \leq t \leq 1 \text {, }
$$

and

$$
\begin{aligned}
\frac{\partial h_{2}(t, s)}{\partial t} & =\frac{(\alpha-1) t^{\alpha-2}}{A}\left((1-s)^{\alpha-\beta-1}-A\left(1-\frac{s}{t}\right)^{\alpha-2}\right) \\
& \geq \frac{(\alpha-1) t^{\alpha-2}}{A}\left((1-s)^{\alpha-\beta-1}-A(1-s)^{\alpha-2}\right) \\
& =\frac{(\alpha-1) t^{\alpha-2}(1-s)^{\alpha-2}}{A}\left((1-s)^{1-\beta}-A\right) \\
& =\frac{(\alpha-1)(t(1-s))^{\alpha-2}}{A}\left((1-s)^{1-\beta}+b \xi^{\alpha-\beta-1}-1\right) \\
& \geq \frac{(\alpha-1)(t(1-s))^{\alpha-2}}{A}\left((1-s)^{1-\beta}-1\right) \geq 0 .
\end{aligned}
$$

So we have $\frac{\partial h_{2}(t, s)}{\partial t} \geq 0$, and thus $g_{2}(t, s)$ is increasing with respect to $t$ on $[s, 1]$.

Then we can conclude that $G(t, s)$ is increasing with respect to $t$ for $t \in[0,1]$. Hence, $G(t, s) \leq G(1, s)$ for $s, t \in[0,1]$.

On the other hand, we know that

$$
\begin{aligned}
\min _{\xi \leq t \leq 1} G(t, s) & = \begin{cases}\min _{\xi \leq t \leq 1}\left\{g_{1}(t, s), g_{3}(t, s)\right\}, & 0 \leq s \leq \xi \\
\min _{\xi \leq t \leq 1}\left\{g_{2}(t, s), g_{4}(t, s)\right\}, & \xi \leq s \leq 1\end{cases} \\
& = \begin{cases}g_{1}(\xi, s), & 0 \leq s \leq \xi \\
g_{2}(\xi, s), & \xi \leq s \leq 1 .\end{cases}
\end{aligned}
$$

Let

$$
\gamma(s) \leq \begin{cases}\frac{g_{1}(\xi, s)}{G(1, s)}, & 0<s \leq \xi \\ \frac{g_{2}(\xi, s)}{G(1, s)}, & \xi \leq s<1\end{cases}
$$


where

$$
\begin{aligned}
G(1, s) & = \begin{cases}g_{1}(1, s), & 0 \leq s \leq \xi, \\
g_{2}(1, s), & \xi \leq s \leq 1\end{cases} \\
& = \begin{cases}\frac{(1-s)^{\alpha-\beta-1}-b(\xi-s)^{\alpha-\beta-1}-A(1-s)^{\alpha-1}}{A \Gamma(\alpha)}, & 0 \leq s \leq \xi, \\
\frac{(1-s)^{\alpha-\beta-1}-A(1-s)^{\alpha-1}}{A \Gamma(\alpha)}, & \xi \leq s \leq 1 .\end{cases}
\end{aligned}
$$

Therefore, we have

$$
\gamma(s)=\xi^{\alpha-1} \in(0,1)
$$

Then

$$
\min _{\xi \leq t \leq 1} G(t, s) \geq \gamma(s) \max _{0 \leq t \leq 1} G(t, s)=\xi^{\alpha-1} G(1, s), \quad 0<s<1 .
$$

The proof is completed.

Lemma 3.3 Assume that $\left(\mathrm{H}_{1}\right)-\left(\mathrm{H}_{2}\right)$ hold. Then the operators $T: K \rightarrow K$ and $L: K \rightarrow K$ are completely continuous.

Proof Since $a(t) \in L^{1}(0,1)$ is nonnegative, $G(t, s)>0$ is continuous on $[0,1] \times[0,1]$, and $f:[0,1] \times[0, \infty] \rightarrow[0, \infty)$ is a continuous function, it is easy to see that $\operatorname{Tu}(t) \geq 0$. So $T: K \rightarrow K$ and $T$ are continuous.

Let $\Phi \subset K$ be bounded, i.e., there exists a positive constant $M$ such that $f(t, u) \leq M$ for all $t \in[0,1], u \in \Phi$. Then

$$
\begin{aligned}
|T u(t)|= & -\int_{0}^{t} \frac{1}{\Gamma(\alpha)} a(s)(t-s)^{\alpha-1} f(s, u(s)) d s+\frac{t^{\alpha-1}}{A \Gamma(\alpha)} \int_{0}^{1} a(s)(1-s)^{\alpha-\beta-1} f(s, u(s)) d s \\
& -\frac{b t^{\alpha-1}}{A \Gamma(\alpha)} \int_{0}^{\xi} a(s)(\xi-s)^{\alpha-\beta-1} f(s, u(s)) d s \mid \\
\leq & -\int_{0}^{t} \frac{1}{\Gamma(\alpha)} a(s)(t-s)^{\alpha-1} f(s, u(s)) d s \mid \\
& +\left|\frac{t^{\alpha-1}}{A \Gamma(\alpha)} \int_{0}^{1} a(s)(1-s)^{\alpha-\beta-1} f(s, u(s)) d s\right| \\
& +\left|-\frac{b t^{\alpha-1}}{A \Gamma(\alpha)} \int_{0}^{\xi} a(s)(\xi-s)^{\alpha-\beta-1} f(s, u(s)) d s\right| \\
& \leq \frac{M}{\Gamma(\alpha)} \int_{0}^{t} a(s)(t-s)^{\alpha-1} d s+\frac{M t^{\alpha-1}}{A \Gamma(\alpha)} \int_{0}^{1} a(s)(1-s)^{\alpha-\beta-1} d s \\
& +\frac{b M t^{\alpha-1}}{A \Gamma(\alpha)} \int_{0}^{\xi} a(s)(\xi-s)^{\alpha-\beta-1} d s \\
\leq & \frac{M}{\Gamma(\alpha)} \int_{0}^{1} a(s) d s+\frac{M}{A \Gamma(\alpha)} \int_{0}^{1} a(s) d s+\frac{b M}{A \Gamma(\alpha)} \int_{0}^{1} a(s) d s \\
= & \frac{M(A+1+b)}{A \Gamma(\alpha)} \int_{0}^{1} a(s) d s .
\end{aligned}
$$


Thus,

$$
\|T u\| \leq \frac{M(A+1+b)}{A \Gamma(\alpha)} \int_{0}^{1} a(s) d s<\infty
$$

for all $u \in \Phi$. Hence, $\{T u, u \in \Phi\}$ is bounded.

On the other hand, for $0 \leq t_{1}<t_{2} \leq 1, u \in \Phi$, we have

$$
\begin{aligned}
& \left|T u\left(t_{2}\right)-T u\left(t_{1}\right)\right|=\mid-\int_{0}^{t_{2}} \frac{1}{\Gamma(\alpha)} a(s)\left(t_{2}-s\right)^{\alpha-1} f(s, u(s)) d s \\
& +\frac{t_{2}^{\alpha-1}}{A \Gamma(\alpha)} \int_{0}^{1} a(s)(1-s)^{\alpha-\beta-1} f(s, u(s)) d s \\
& -\frac{b t_{2}^{\alpha-1}}{A \Gamma(\alpha)} \int_{0}^{\xi} a(s)(\xi-s)^{\alpha-\beta-1} f(s, u(s)) d s \\
& +\int_{0}^{t_{1}} \frac{1}{\Gamma(\alpha)} a(s)\left(t_{1}-s\right)^{\alpha-1} f(s, u(s)) d s \\
& -\frac{t_{1}^{\alpha-1}}{A \Gamma(\alpha)} \int_{0}^{1} a(s)(1-s)^{\alpha-\beta-1} f(s, u(s)) d s \\
& +\frac{b t_{1}^{\alpha-1}}{A \Gamma(\alpha)} \int_{0}^{\xi} a(s)(\xi-s)^{\alpha-\beta-1} f(s, u(s)) d s \\
& \leq \mid \int_{0}^{t_{1}} \frac{1}{\Gamma(\alpha)} a(s)\left(t_{1}-s\right)^{\alpha-1} f(s, u(s)) d s \\
& -\int_{0}^{t_{2}} \frac{1}{\Gamma(\alpha)} a(s)\left(t_{2}-s\right)^{\alpha-1} f(s, u(s)) d s \mid \\
& +\mid \frac{t_{2}^{\alpha-1}}{A \Gamma(\alpha)} \int_{0}^{1} a(s)(1-s)^{\alpha-\beta-1} f(s, u(s)) d s \\
& -\frac{t_{1}^{\alpha-1}}{A \Gamma(\alpha)} \int_{0}^{1} a(s)(1-s)^{\alpha-\beta-1} f(s, u(s)) d s \\
& +\mid \frac{b t_{1}^{\alpha-1}}{A \Gamma(\alpha)} \int_{0}^{\xi} a(s)(\xi-s)^{\alpha-\beta-1} f(s, u(s)) d s \\
& -\frac{b t_{2}^{\alpha-1}}{A \Gamma(\alpha)} \int_{0}^{\xi} a(s)(\xi-s)^{\alpha-\beta-1} f(s, u(s)) d s \mid \\
& \leq\left|\int_{0}^{t_{1}} \frac{1}{\Gamma(\alpha)} a(s)\left(\left(t_{1}-s\right)^{\alpha-1}-\left(t_{2}-s\right)^{\alpha-1}\right) f(s, u(s)) d s\right| \\
& +\left|\int_{t_{1}}^{t_{2}} \frac{1}{\Gamma(\alpha)} a(s)\left(t_{2}-s\right)^{\alpha-1} f(s, u(s)) d s\right| \\
& +\frac{M\left(t_{2}^{\alpha-1}-t_{1}^{\alpha-1}\right)}{A \Gamma(\alpha)} \int_{0}^{1} a(s) d s+\frac{b M\left(t_{2}^{\alpha-1}-t_{1}^{\alpha-1}\right)}{A \Gamma(\alpha)} \int_{0}^{1} a(s) d s \\
& \leq \frac{M}{\Gamma(\alpha)}\left|\int_{0}^{t_{1}} a(s)\left(\left(t_{1}-s\right)^{\alpha-1}-\left(t_{2}-s\right)^{\alpha-1}\right) d s\right| \\
& +\frac{M}{\Gamma(\alpha)}\left|\int_{t_{1}}^{t_{2}} a(s)\left(t_{2}-s\right)^{\alpha-1} d s\right| \\
& +\frac{M(1+b)\left(t_{2}^{\alpha-1}-t_{1}^{\alpha-1}\right)}{A \Gamma(\alpha)} \int_{0}^{1} a(s) d s
\end{aligned}
$$




$$
\begin{aligned}
\leq & \frac{M\left(t_{2}-t_{1}\right)}{\Gamma(\alpha)} \int_{0}^{t_{1}} a(s) d s+\frac{M}{\Gamma(\alpha)}\left|\int_{t_{1}}^{t_{2}} a(s)\left(t_{2}-s\right)^{\alpha-1} d s\right| \\
& +\frac{M(1+b)\left(t_{2}^{\alpha-1}-t_{1}^{\alpha-1}\right)}{A \Gamma(\alpha)} \int_{0}^{1} a(s) d s .
\end{aligned}
$$

Since $a(t) \in L^{1}(0,1)$, by the integration of Cauchy's test for convergence and the uniform continuity of $t^{\alpha}, t^{\alpha-1}$ on $[0,1]$, we can get that $\{T u, u \in \Phi\}$ is equicontinuous. By the ArzelaAscoli theorem we conclude that $T: K \rightarrow K$ is a completely continuous operator.

By the same method we can get that $L: K \rightarrow K$ is a completely continuous operator. The proof is completed.

Now we use the Banach contraction mapping principle to prove that the boundary value problem (1.1)-(1.2) has a unique solution on $[0,1]$.

Theorem 3.1 Assume that $\left(\mathrm{H}_{1}\right)$ and $\left(\mathrm{H}_{2}\right)$ hold and there exists a constant $\ell$ such that

$$
|f(t, x)-f(t, y)| \leq \ell|x-y|
$$

for almost every $t \in[0,1]$ and all $x, y \in[0, \infty)$.

If

$$
0<\ell \int_{0}^{1} G(1, s) a(s) d s<1
$$

then the boundary value problem (1.1)-(1.2) has a unique positive solution on $[0,1]$.

Proof By Lemma 3.1, $T: K \rightarrow K$. Moreover, for any $u, v \in K$, we deduce

$$
\begin{aligned}
|T u(t)-T v(t)| & =\left|\int_{0}^{1} G(t, s) a(s)(f(s, u(s))-f(s, v(s))) d s\right| \\
& \leq \int_{0}^{1} G(t, s) a(s)|f(s, u(s))-f(s, v(s))| d s \\
& \leq \ell \int_{0}^{1} G(1, s) a(s)|u(s)-v(s)| d s \\
& \leq \ell \int_{0}^{1} G(1, s) a(s) d s\|u-v\| .
\end{aligned}
$$

This implies that

$$
\|T u-T v\| \leq \alpha\|u-v\|
$$

where $\alpha=\ell \int_{0}^{1} G(1, s) a(s) d s \in(0,1)$. So $T$ is a contraction mapping. Hence, by the Banach contraction mapping principle the boundary value problem (1.1)-(1.2) has a unique positive solution on $[0,1]$. The proof is completed.

Remark 3.2 For $a \in C[0,1]$, the integral condition reduces to $\ell \sigma\|a\|<1$, where $\sigma=$ $\int_{0}^{1} G(1, s) d s$ is the exact value. 
Now we study the existence of solutions for the boundary value problem (1.1)-(1.2) by the fixed point index theory.

Lemma 3.4 Assume that $\left(\mathrm{H}_{1}\right)-\left(\mathrm{H}_{2}\right)$ hold. Then the spectral radius of the operator $L$ is positive, that is, $r(L)>0$.

Proof Take $u(t)=t^{\alpha-1} \in E$. Then $\|u\|=1$.

We have

$$
\begin{aligned}
L u(t)= & -\int_{0}^{t} \frac{1}{\Gamma(\alpha)} a(s)(t-s)^{\alpha-1} u(s) d s+\frac{t^{\alpha-1}}{A \Gamma(\alpha)} \int_{0}^{1} a(s)(1-s)^{\alpha-\beta-1} u(s) d s \\
& -\frac{b t^{\alpha-1}}{A \Gamma(\alpha)} \int_{0}^{\xi} a(s)(\xi-s)^{\alpha-\beta-1} u(s) d s \\
= & -\frac{t^{\alpha-1}}{\Gamma(\alpha)} \int_{0}^{t} a(s)\left(1-\frac{s}{t}\right)^{\alpha-1} s^{\alpha-1} d s+\frac{t^{\alpha-1}}{A \Gamma(\alpha)} \int_{0}^{1} a(s)(1-s)^{\alpha-\beta-1} s^{\alpha-1} d s \\
& -\frac{b t^{\alpha-1} \xi^{\alpha-\beta-1}}{A \Gamma(\alpha)} \int_{0}^{\xi} a(s)\left(1-\frac{s}{\xi}\right)^{\alpha-\beta-1} s^{\alpha-1} d s \\
= & t^{\alpha-1}\left(-\frac{1}{\Gamma(\alpha)} \int_{0}^{t} a(s)\left(1-\frac{s}{t}\right)^{\alpha-1} s^{\alpha-1} d s+\frac{1}{A \Gamma(\alpha)} \int_{0}^{1} a(s)(1-s)^{\alpha-\beta-1} s^{\alpha-1} d s\right. \\
& \left.-\frac{b \xi^{\alpha-\beta-1}}{A \Gamma(\alpha)} \int_{0}^{\xi} a(s)\left(1-\frac{s}{\xi}\right)^{\alpha-\beta-1} s^{\alpha-1} d s\right) \\
> & t^{\alpha-1}\left(-\frac{1}{\Gamma(\alpha)} \int_{0}^{1} a(s)\left(1-\frac{s}{t}\right)^{\alpha-1} s^{\alpha-1} d s+\frac{1}{A \Gamma(\alpha)} \int_{0}^{1} a(s)(1-s)^{\alpha-\beta-1} s^{\alpha-1} d s\right. \\
& \left.-\frac{b \xi^{\alpha-\beta-1}}{A \Gamma(\alpha)} \int_{0}^{1} a(s)\left(1-\frac{s}{\xi}\right)^{\alpha-\beta-1} s^{\alpha-1} d s\right) \\
> & t^{\alpha-1}\left(-\frac{1}{\Gamma(\alpha)} \int_{0}^{1} a(s)(1-s)^{\alpha-1} s^{\alpha-1} d s+\frac{1}{A \Gamma(\alpha)} \int_{0}^{1} a(s)(1-s)^{\alpha-\beta-1} s^{\alpha-1} d s\right. \\
& \left.-\frac{b \xi^{\alpha-\beta-1}}{A \Gamma(\alpha)} \int_{0}^{1} a(s)(1-s)^{\alpha-\beta-1} s^{\alpha-1} d s\right) \\
= & t^{\alpha-1}\left(-\frac{1}{\Gamma(\alpha)} \int_{0}^{1} a(s)(1-s)^{\alpha-1} s^{\alpha-1} d s+\frac{1}{\Gamma(\alpha)} \int_{0}^{1} a(s)(1-s)^{\alpha-\beta-1} s^{\alpha-1} d s\right) \\
>0 . &
\end{aligned}
$$

Since $L: K \rightarrow K$, according to the monotonicity of $L$ and $\left(\mathrm{H}_{2}\right)$, we deduce

$$
\begin{aligned}
L^{2} u(t) & =L(L u(t))>L\left(l t^{\alpha-1}\right) \\
& >l L\left(t^{\alpha-1}\right)>l^{2} t^{\alpha-1} .
\end{aligned}
$$

Repeating the process gives $L^{n} u(t)>l^{n} t^{\alpha-1}$. So we get $\left\|L^{n}\right\|>l^{n}$. Hence,

$$
\left\|L^{n}\right\|^{\frac{1}{n}}>l, \quad r(L)=\lim _{n \rightarrow \infty}\left\|L^{n}\right\|^{\frac{1}{n}}>l>0 .
$$

The proof is completed. 
Define

$$
\begin{aligned}
& f_{0}=\liminf _{u \rightarrow 0^{+}} \min _{t \in[0,1]} \frac{f(t, u)}{u}, \\
& f^{\infty}=\limsup _{u \rightarrow \infty} \max _{t \in[0,1]} \frac{f(t, u)}{u}, \\
& K_{c}=\{x \in K:\|x\|<c\}, \\
& r(L)=\frac{1}{\mu}, \quad \mu \in R^{+} .
\end{aligned}
$$

Lemma 3.5 Assume that $\left(\mathrm{H}_{1}\right)-\left(\mathrm{H}_{2}\right)$ hold and $\mu<f_{0} \leq \infty$. Then there exists $\rho_{0}>0$ such that for $\rho \in\left(0, \rho_{0}\right]$, if $u \neq T u, u \in \partial K_{\rho}$, then $i\left(T, K_{\rho}, K\right)=0$.

Proof It follows from $\mu<f_{0}$ that there exist $\varepsilon>0$ and $\rho_{0}>0$ such that for $t \in[0,1]$ and $0 \leq u \leq \rho_{0}$

$$
f(t, u) \geq(\mu+\varepsilon) u
$$

For $0<\rho<\rho_{0}$, assume that $u \neq T u, u \in \partial K_{\rho}$. By Lemma 2.5 and Lemma 2.6(i) we need only to prove that

$$
u \neq T u+\lambda \varphi, \quad \lambda>0
$$

where $\varphi \in K \backslash\{0\}$ with $L \varphi=r(L) \varphi$.

Otherwise, there exist $u_{0} \in \partial K_{\rho}$ and $\lambda_{0}>0$ such that

$$
u_{0}=T u_{0}+\lambda_{0} \varphi
$$

Then, $u_{0} \geq T u_{0}$ and $u_{0} \geq \lambda_{0} \varphi$. By (3.1) we get that

$$
T u_{0}(t)=\int_{0}^{1} G(t, s) a(s) f\left(s, u_{0}(s)\right) d s \geq(\mu+\varepsilon) L u_{0}(t)
$$

Considering $u_{0} \geq \lambda_{0} \varphi$, we have

$$
L u_{0} \geq \lambda_{0} L \varphi
$$

For $L \varphi=r(L) \varphi,(\mu+\varepsilon) r(L)>1$, so that $(\mu+\varepsilon) r(L) \varphi>\varphi$. So we can conclude

$$
T u_{0} \geq(\mu+\varepsilon) \lambda_{0} L \varphi>\lambda_{0} \varphi
$$

Together with (3.2), we have $u_{0} \geq 2 \lambda_{0} \varphi$. By (3.3) we have $T u_{0} \geq 2 \lambda_{0} \varphi$. So $u_{0} \geq 3 \lambda_{0} \varphi$. $\operatorname{Re}$ peating this process, we get that $u_{0} \geq n \lambda_{0} \varphi$, so that we have $\left\|u_{0}\right\| \geq n \lambda_{0}\|\varphi\| \rightarrow \infty, n \rightarrow \infty$. This is a contradiction.

It follows from Lemma 2.6(i) that $i\left(T, K_{\rho}, K\right)=0, \rho \in\left(0, \rho_{0}\right]$. The proof is completed. 
Lemma 3.6 Assume that $\left(\mathrm{H}_{1}\right)-\left(\mathrm{H}_{2}\right)$ hold and $0 \leq f^{\infty}<\mu$. Then there exists $\tau_{0}>0$ such that for each $\tau>\tau_{0}$, if $\lambda u \neq T u, u \in \partial K_{\tau}$, then $i\left(T, K_{\tau}, K\right)=1$.

Proof Let $\epsilon>0$ satisfy $f^{\infty}<\mu-\epsilon$. Then there exists $\tau_{1}>0$ such that for $u>\tau_{1}$ and $t \in[0,1]$,

$$
f(t, u) \leq(\mu-\epsilon) u
$$

Set $\Psi(t)=\max _{u \in\left[0, \tau_{1}\right]} f(t, u)$. Then, for all $u \in R^{+}$and $t \in[0,1]$, we have

$$
f(t, u) \leq(\mu-\epsilon) u+\Psi(t) .
$$

Let

$$
F=\left\|\int_{0}^{1} a(s) G(t, s) \Psi(s) d s\right\|, \quad \tau_{0}=\left\|\frac{F}{\mu-\epsilon}\left(\frac{I}{\mu-\epsilon}-L\right)^{-1}\right\| .
$$

Take $\tau>\tau_{0}$. We will show that $\lambda u \neq T u$ for all $u \in \partial K_{\tau}$ and $\lambda \geq 1$.

Otherwise, there exist $u_{0} \in \partial K_{\tau}$ and $\lambda_{0} \geq 1$ such that

$$
T u_{0}=\lambda_{0} u_{0} .
$$

Together with (3.7), we have

$$
u_{0} \leq \lambda_{0} u_{0}=T u_{0} \leq(\mu-\epsilon) L u_{0}+F
$$

Then $\left(\frac{I}{\mu-\epsilon}-L\right) u_{0}(t) \leq \frac{F}{\mu-\epsilon}$ for $t \in[0,1]$. So we deduce $\frac{F}{\mu-\epsilon}-\left(\frac{I}{\mu-\epsilon}-L\right) u_{0} \in K$. It follows from $L(K) \subset K$ that $u_{0}(t) \leq \frac{F}{\mu-\epsilon}\left(\frac{I}{\mu-\epsilon}-L\right)^{-1}$ for $t \in[0,1]$. Therefore, we have $\left\|u_{0}\right\| \leq \tau_{0}<\tau$. This is a contradiction. So we can conclude that

$$
T u \neq \lambda u
$$

for all $u \in \partial K_{\tau}$ and $\lambda \geq 1$.

By Lemma 2.6(ii) we get that $i\left(T, K_{\tau}, K\right)=1$ for each $\tau_{0}<\tau$. The proof is completed.

Theorem 3.2 Suppose that $\left(\mathrm{H}_{1}\right)-\left(\mathrm{H}_{2}\right)$ hold, $\mu<f_{0} \leq \infty$, and $0 \leq f^{\infty}<\mu$. Then the boundary value problem (1.1)-(1.2) has at least one positive solution on $[0,1]$.

Proof It follows from $\mu<f_{0} \leq \infty$ and Lemma 3.5 that there exists $0<\rho<\tau$ such that either there exists $u \in \partial K_{\rho}$ with $u=T u$ or $i\left(T, K_{\rho}, K\right)=0$. By $0 \leq f^{\infty}<\mu$ and Lemma 3.6 there exists $\tau>0$ such that $i\left(T, K_{\tau}, K\right)=1$. So we can conclude that $T$ has a fixed point $u \in K$ with $\rho<\|u\|<\tau$ by the properties of index. Hence, the boundary value problem (1.1)-(1.2) has at least one positive solution on $[0,1]$. The proof is completed.

Now we study the multiplicity of solutions for the boundary value problem (1.1)-(1.2) by the Leggett-Williams fixed point theorem.

Theorem 3.3 Assume that $\left(\mathrm{H}_{1}\right)-\left(\mathrm{H}_{2}\right)$ hold. Suppose that there exist constants $0<a<b_{1}<c$ such that the following assumptions hold: 
(i) $f(t, u)<M a,(t, u) \in[0,1] \times[0, a]$,

(ii) $f(t, u) \leq M c,(t, u) \in[0,1] \times[0, c]$,

(iii) $f(t, u) \geq N b_{1},(t, u) \in[\xi, 1] \times\left[b_{1}, c\right]$,

where $\gamma(s) \in(0,1), M=\left(\int_{0}^{1} a(s) G(1, s) d s\right)^{-1}$, and $N=\left(\int_{\xi}^{1} a(s) \gamma(s) G(1, s) d s\right)^{-1}$. Then the boundary value problem (1.1)-(1.2) has at least three positive solutions $u_{1}, u_{2}$, and $u_{3}$ with

$$
\left\|u_{1}\right\|<a, \quad b_{1}<\phi\left(u_{2}\right)<\left\|u_{2}\right\| \leq c, \quad \text { and } \quad a<\left\|u_{3}\right\|, \quad \phi\left(u_{3}\right)<b_{1} .
$$

Proof If $u \in \bar{K}_{c}$, then $\|u\| \leq c$. So $0 \leq u(t) \leq c, t \in[0,1]$. By condition (ii) we have

$$
\begin{aligned}
|T u(t)| & =\mid \int_{0}^{1} G(t, s) a(s)(f(s, u(s)) d s \mid \\
& \leq \int_{0}^{1} G(1, s) a(s) M c d s \\
& =M c \int_{0}^{1} a(s) G(1, s) d s=c,
\end{aligned}
$$

which implies that $\|T u\| \leq c, u \in \bar{K}_{c}$. Hence, $T: \bar{K}_{c} \rightarrow \bar{K}_{c}$. In view of Lemma 3.2, $T: \bar{K}_{c} \rightarrow$ $\bar{K}_{c}$ is completely continuous.

Next, by using the analogous argument it follows from condition (i) that if $u \in \bar{K}_{a}$, then $\|T u\|<a$.

Choose $u(t)=\frac{b_{1}+c}{2}, t \in[0,1]$. It is easy to see that $u(t) \in K\left(\phi, b_{1}, c\right), \phi\left(\frac{b_{1}+c}{2}\right)=\frac{b_{1}+c}{2}>b_{1}$. Therefore, $\left\{u \in K\left(\phi, b_{1}, c\right) \mid \phi(u)>b_{1}\right\} \neq \emptyset$.

On the other hand, if $u \in K\left(\phi, b_{1}, c\right)$, then $b_{1} \leq u(t) \leq c, t \leq[\xi, 1]$. By condition (iii) we have

$$
f(t, u(t)) \geq N b_{1}
$$

Hence,

$$
\begin{aligned}
\phi(T u) & =\min _{\xi \leq t \leq 1}|T u(t)|=\min _{\xi \leq t \leq 1} \mid \int_{0}^{1} G(t, s) a(s)(f(s, u(s)) d s \mid \\
& \geq \min _{\xi \leq t \leq 1} \int_{0}^{1} G(t, s) a(s) N b_{1} d s \\
& >\int_{\xi}^{1} \gamma(s) G(1, s) a(s) N b_{1} d s \\
& =N b_{1} \int_{\xi}^{1} a(s) \gamma(s) G(1, s) d s=b_{1},
\end{aligned}
$$

which implies that $\phi(T u)>b_{1}$ for $u \in K\left(\phi, b_{1}, c\right)$.

In conclusion, by Lemma 2.7 and Remark 2.1 the boundary value problem (1.1)-(1.2) has at least three positive solution $u_{1}, u_{2}$, and $u_{3}$ with

$$
\left\|u_{1}\right\|<a, \quad b_{1}<\phi\left(u_{2}\right)<\left\|u_{2}\right\| \leq c, \quad \text { and } \quad a<\left\|u_{3}\right\|, \quad \phi\left(u_{3}\right)<b_{1} .
$$

The proof is completed. 


\section{Examples}

In this section, we present some examples to illustrate our main results.

Example 4.1 Consider the following boundary value problem:

$$
\begin{aligned}
& D_{0^{+}}^{\frac{5}{2}} u(t)+a(t)\left(\frac{e^{t}}{2\left(1+e^{t}\right)(1+u)}+\sin ^{2} t+1\right)=0, \quad 0<t<1, \\
& u(0)=0, \quad D_{0^{+}}^{\frac{3}{2}} u(0)=0, \quad D_{0^{+}}^{\frac{3}{2}} u(1)=b D_{0^{+}}^{\frac{3}{2}} u(\xi),
\end{aligned}
$$

where $b=\frac{1}{100}$ and $\xi=\frac{1}{10}$.

Here

$$
\begin{aligned}
& \alpha=\frac{5}{2}, \quad \beta=\frac{3}{2}, \quad a(t)=\frac{(1-t)^{\frac{1}{2}}}{t^{\frac{3}{2}}}, \quad f(t, u)=\frac{e^{t}}{2\left(1+e^{t}\right)(1+u)}+\sin ^{2} t+1, \\
& (t, u) \in[0,1] \times[0, \infty], \ell=\frac{1}{4} .
\end{aligned}
$$

It is clear that $|f(t, u)-f(t, v)| \leq \ell|u-v|$ for $(t, u),(t, v) \in[0,1] \times[0, \infty]$.

Since $a(t)$ is singular at $t=0$, by simple calculation we have

$$
\begin{aligned}
0 & <\ell \int_{0}^{1} G(1, s) a(s) d s \leq \ell \int_{0}^{1} \frac{(1-s)^{\frac{1}{2}}(1-s)^{\alpha-\beta-1}-\left(1-b \xi^{\alpha-\beta-1}\right)(1-s)^{\alpha-1}(1-s)^{\frac{1}{2}}}{s^{\frac{3}{2}}\left(1-b \xi^{\alpha-\beta-1}\right) \Gamma(\alpha)} \\
& \approx 0.1148<1 .
\end{aligned}
$$

By Theorem 3.1 we see that the boundary value problem (4.1)-(4.2) has a unique solution.

Example 4.2 Consider the following boundary value problem:

$$
\begin{aligned}
& D_{0^{+}}^{\frac{5}{2}} u(t)+a(t) f(t, u)=0, \quad 0<t<1, \\
& u(0)=0, \quad D_{0^{+}}^{\frac{3}{2}} u(0)=0, \quad D_{0^{+}}^{\frac{3}{2}} u(1)=b D_{0^{+}}^{\frac{3}{2}} u(\xi),
\end{aligned}
$$

where

$$
f(t, u)= \begin{cases}u^{\frac{1}{2}}+t^{\frac{1}{2}}, & u \in[0,1], t \in[0,1] \\ u^{\frac{1}{3}}+t^{\frac{1}{2}}, & u \in(1, \infty), t \in[0,1]\end{cases}
$$

and $b=\frac{1}{100}, \xi=\frac{1}{10}$.

Here $\alpha=\frac{5}{2}, \beta=\frac{3}{2}, a(t)=\frac{1}{1-t}$. It is easy to see that $\left(\mathrm{H}_{1}\right)$ and $\left(\mathrm{H}_{2}\right)$ are satisfied. By simple calculation we can conclude that

$$
f_{0}=\infty, \quad f^{\infty}=0 .
$$

Hence, by Theorem 3.2 we obtain that the boundary value problem (4.3)-(4.4) has at least a solution. 
Example 4.3 Consider the following boundary value problem:

$$
\begin{aligned}
& D_{0^{+}}^{\frac{5}{2}} u(t)+a(t) f(t, u)=0, \quad 0<t<1, \\
& u(0)=0, \quad D_{0^{+}}^{\frac{3}{2}} u(0)=0, \quad D_{0^{+}}^{\frac{3}{2}} u(1)=b D_{0^{+}}^{\frac{3}{2}} u(\xi),
\end{aligned}
$$

where

$$
f(t, u)= \begin{cases}9 u^{2}+\frac{t}{100}, & u \in[0,1], t \in[0,1] \\ 9, & u>1, t \in[0,1]\end{cases}
$$

and $b=\frac{1}{5}, \xi=\frac{1}{2}$.

Here $\alpha=\frac{5}{2}, \beta=\frac{3}{2}, a(t)=1$. By simple calculation we have

$$
\begin{aligned}
& A=1-b \xi^{\alpha-\beta-1}=0.8 \\
& \int_{0}^{1} a(s) G(1, s) d s=\int_{0}^{1} \frac{(1-s)^{\alpha-\beta-1}-\left(1-b \xi^{\alpha-\beta-1}\right)(1-s)^{\alpha-1}}{\left(1-b \xi^{\alpha-\beta-1}\right) \Gamma(\alpha)} d s \approx 0.6394
\end{aligned}
$$

and

$$
\int_{\xi}^{1} \gamma(s) a(s) G(1, s) d s=\int_{\frac{1}{2}}^{1} g_{2}(\xi, s) d s \approx 0.1130
$$

Hence, $M=\left(\int_{0}^{1} a(s) G(1, s) d s\right)^{-1} \approx 1.5639$ and $N=\left(\int_{\xi}^{1} \gamma(s) a(s) G(1, s) d s\right)^{-1} \approx 8.8462$.

Choosing $a=\frac{1}{6}, b_{1}=1$, and $c=6$, we have

$$
\begin{aligned}
& f(t, u)=9 u^{2}+\frac{t}{100} \leq 0.26<M a=0.26065, \quad(t, u) \in[0,1] \times\left[0, \frac{1}{6}\right] \\
& f(t, u)=9 u^{2}+\frac{t}{100} \leq 9.01<M c=9.3834, \quad(t, u) \in[0,1] \times[0,1] \\
& f(t, u)=9 \leq M c=9.3834, \quad(t, u) \in[0,1] \times(1,6] \\
& f(t, u)=9 \geq N b_{1}=8.8462, \quad(t, u) \in\left[\frac{1}{2}, 1\right] \times(1,6] .
\end{aligned}
$$

Hence, by Theorem 3.3 we obtain that the boundary value problem (4.5)-(4.6) has at least three positive solutions $u_{1}, u_{2}$, and $u_{3}$ such that

$$
\left\|u_{1}\right\|<\frac{1}{6}, \quad 1<\phi\left(u_{2}\right)<\left\|u_{2}\right\| \leq 6, \quad \text { and } \quad \frac{1}{6}<\left\|u_{3}\right\|, \quad \phi\left(u_{3}\right)<1 .
$$

\section{Conclusion}

In this paper, we systematically study the existence, uniqueness, and multiplicity of positive solutions for a class of Riemann-Liouville fractional differential equations with threepoint boundary conditions. By the properties of Green function, we establish the existence and uniqueness of single positive solutions to the three-point boundary value problems (1.1)-(1.2) by the Banach contraction mapping principle and the fixed point index theory, 
and we investigate the existence of multiple positive solutions for (1.1)-(1.2) by the LeggettWilliams fixed point theorem. As applications, examples are presented to illustrate the main results.

We propose some techniques to prove the completely continuity of operators and the properties of the Green function that need not adding more preconditions. With the elevation of order, the properties of the Green function happen to change. We use these new properties to obtain more general results. We investigate the fractional differential equations without many preconditions by the fixed point index theory and obtain the existence of single positive solutions.

\section{Competing interests}

The authors declare that they have no competing interests.

\section{Authors' contributions}

The authors declare that the study was realized in collaboration with the same responsibility. All authors read and approved the final manuscript.

\section{Author details}

'School of Mathematical Sciences, University of Jinan, Jinan, Shandong 250022, P.R. China. ${ }^{2}$ School of Control Science and Engineering, University of Jinan, Jinan, Shandong 250022, P.R. China.

\section{Acknowledgements}

The authors sincerely thank the reviewers for their valuable suggestions and useful comments that have led to the present improved version of the original manuscript. This research is supported by the Natural Science Foundation of China $(61374074,11571202)$ and by the Shandong Provincial Natural Science Foundation (ZR2013AL003).

Received: 15 May 2015 Accepted: 28 November 2015 Published online: 24 December 2015

\section{References}

1. Oldham, K, Spanier, J: The Fractional Calculus. Academic Press, New York (1974)

2. Miller, K, Ross, B: An Introduction to the Fractional Calculus and Fractional Differential Equation. Wiley, New York (1993)

3. Samko, S, Kilbas, A, Marichev, O: Fractional Integral and Derivative, Theory and Applications. Gordon \& Breach, Yverdon (1993)

4. Podlubny, I: Fractional Differential Equations. Academic Press, San Diego (1999)

5. Kilbas, A, Srivastava, H, Trujillo, J: Theory and Applications of Fractional Differential Equations. North-Holland, Amsterdam (2006)

6. Zeidler, E: Nonlinear Functional Analysis and Its Applications I: Fixed-Point Theorems. Springer, Berlin (1985)

7. Ahmad, B, Ntouyas, SK: On higher-order sequential fractional differential inclusions with nonlocal three-point boundary conditions. Abstr. Appl. Anal. 2014, Article ID 659405 (2014)

8. Bai, Z, Lü, H: Positive solutions for boundary value problem of nonlinear fractional equation. J. Math. Anal. Appl. 311 , 495-505 (2005)

9. Zhang, S: Positive solutions for boundary value problems of nonlinear fractional differential equations. Electron. J. Differ. Equ. 2006, 36 (2006)

10. Ahmad, B, Agarwal, RP: Some new versions of fractional boundary value problems with slit-strips conditions. Bound. Value Probl. 2014, Article ID 175 (2014)

11. Bai, Z: On positive solutions of a nonlocal fractional boundary value problem. Nonlinear Anal. 72, $916-924$ (2010)

12. Zhai, C, Xu, L: Properties of positive solutions to a class of four-point boundary value problem of Caputo fractional differential equations with a parameter. Commun. Nonlinear Sci. Numer. Simul. 19, 2820-2827 (2014)

13. Ahmad, B, Nieto, JJ: Riemann-Liouville fractional differential equations with fractional boundary conditions. Fixed Point Theory 13, 329-336 (2012)

14. Zhai, C, Yan, W, Yang, C: A sum operator method for the existence and uniqueness of positive solutions to Riemann-Liouville fractional differential equation boundary value problems. Commun. Nonlinear Sci. Numer. Simul. $18,858-866(2013)$

15. Li, C, Luo, X, Zhou, Y: Existence of positive solutions of the boundary value problem for nonlinear fractional differential equations. Comput. Math. Appl. 59, 1363-1375 (2010)

16. Ahmad, B, Nieto, JJ: A class of differential equations of fractional order with multi-point boundary conditions. Georgian Math. J. 21, 243-248 (2014)

17. Jiang, W, Wang, B, Wang, Z: The existence of positive solutions for multi-point boundary value problems of fractional differential equations. Phys. Proc. 25, 958-964 (2012)

18. Ahmad, B, Alsaedi, A, Al-Hutami, H: Existence of solutions for sequential fractional differential equations with four-point nonlocal fractional integral boundary conditions. Cent. Eur. J. Phys. 11, 1487-1493 (2013)

19. Li, B, Sun, S, Li, Y: Multi-point boundary value problems for a class of Riemann-Liouville fractional differential equations. Adv. Differ. Equ. 2014, Article ID 151 (2014)

20. Ahmad, B, Ntouyas, SK, Alsaedi, A: A study of nonlinear fractional differential equations of arbitrary order with Riemann-Liouville type multistrip boundary conditions. Math. Probl. Eng. 2013, Article ID 320415 (2013) 